\title{
空化泡内外质量交换的唯象模型
}

高贤娴, 陈伟中", 黄威, 徐俊峰, 徐兴华, 刘亚楠, 梁越

南京大学声学研究所, 近代声学教育部重点实验室, 南京 210093

* 联系人, E-mail: wzchen@nju.edu.cn

2008-10-08 收稿, 2008-12-10 接受

国家自然科学基金(批准号: 10434070, 10704037)、教育部重点项目(编号: 103078)和江苏省创新人才项目(编号: BK2007569)资助

Gao X X, Chen W Z, Huang W, et al. A phenomenological model of mass-exchange between the inside and outside of a cavitation bubble. Chinese Science Bulletin, 2009, 54, doi: 10.1007/s11434-009-0156-9

摘要 空化泡内外的质量交换是一个多种交换方式共同作用的综合过程，其中包括气体扩散、气液 相变、化学反应等. 本文提出了一个描述空化泡内外质量交换的唯象模型, 它以空化泡内外压强差 作为质量交换的驱动力. 与已有的具体物理模型相比，具有形式简单、计算量小的特点. 联合 Rayleigh-Plesset 气泡动力学方程, 计算了质量交换动态平衡后的空化泡平衡半径. 结果表明, 平衡 关键词 空化泡 质量交换 平衡半径 半径具有多重性. 另外计算了平衡半径与驱动声压的关系, 并研究了模型参数对其结果的影响. 最 后, 对不同驱动声压下的硫酸中空化泡脉动进行了实验测量, 得到的平衡半径随声压变化的关系 很好地支持了模型计算.

我们知道, 水中含有空气泡, 但通常这些空气泡 很小, 肉眼不能直接观测. 当一定强度的超声波进入 液体之后，在负压作用下，原本不可见的空气泡(空 化核)成长为肉眼可见的微气泡, 这种现象就是超声 空化, 所产生的微气泡就是空化泡. 空化泡在声场作 用下作非线性的膨胀和收缩. 在空化泡塌缩期间, 其 内形成极端高温高压. 当驱动声压进一步增大, 空化 泡在剧烈塌缩时产生发光现象, 即声致发光 ${ }^{[1]}$. 因此, 空化泡动力学的研究是声化学、声致发光等课题研究 的基础.

在理论上, 人们一般采用Rayleigh气泡动力学方 程来描述空化泡的脉动 ${ }^{[2]}$, 其中存在一个描述气泡大 小的重要模型参数——环境半径 $R_{\mathrm{amb}}$ (ambient radius), 它是在环境压力(ambient pressure)下的气泡半径, 也 就是无驱动声压下气泡的静态半径 ${ }^{[3]}$. 环境半径 $R_{\mathrm{amb}}$ 和泡内物质的量 $n$, 可以通过理想气体方程 $P_{0}\left(\frac{4}{3} \pi R_{\mathrm{amb}}^{3}\right)=n R_{\mathrm{g}} T_{0}$ 相互联系, 其中 $P_{0}$ 为环境声压, $T_{0}$ 为环境温度, $R_{\mathrm{g}}$ 是气体常数. 因为一般情况下 $\left(P_{0}, T_{0}\right)$
的状态与标准状态相近, 所以我们可用理想气体方 程近似描述. 人们通常将空化泡看成是一个封闭的 系统, 内部质量数 $n$ 保持不变, 气泡的环境半径 $R_{\mathrm{amb}}$ 为常数, 不随时间变化, 因此也称之为平衡半径 $R_{0}=R_{\mathrm{amb}} . \mathrm{Xu}$ 等人 ${ }^{[4]}$ 通过实验发现, 声致发光的光谱会 随时间发生变化, 表明气泡内物质发生了变化, 并不 是一个完全封闭的系统, 空化泡内部质量数 $n$ 不是不 变的, 对应的环境半径 $R_{\mathrm{amb}}$ 也不是常数, 而是随时间 变化的, 因此我们将它记为 $R_{\mathrm{amb}}(t)$. 实验还发现, 不 管声致发光的起始光谱如何变化, 空化泡的光谱最 终都会达到一个稳定不变的状态. 可见, 气泡内外可 以存在物质交换, 而且最终会演化成平衡状态. 实际 上, 我们在实验中注意到, 无论初始注入的气泡大小 如何, 在一定的驱动声压下能够稳定存在的气泡具 有确定的平衡半径. 这一事实也证明, $R_{\mathrm{amb}}(t)$ 是可变 化的, 但最终会趋向一个稳定的平衡半径 $R_{0}, R_{\mathrm{amb}}(t)$ 和 $R_{0}$ 在可变质量的系统中表达的意义不再相同.

从物理结构上看, 空化泡的壁面是气液界面, 可 以存在多种交换机制, 例如气体扩散 ${ }^{[5]}$ 、气液相变 ${ }^{[6]}$ 
和化学反应 ${ }^{[7 \sim 9]}$ 等. 很多研究人员对质量交换的过程 作过深入的研究. 1996 年Lohse等人 ${ }^{[5]}$ 考虑空化泡内 外的气体扩散, 得到了稳定声致发光的相图. 之后他 们又考虑了氮气相关的化学反应以及氮气和氞气的 扩散, 重新计算了相图, 并提出了氩气精馏理论 ${ }^{[7,8]}$, 其结果得到了实验 ${ }^{[10,11]}$ 的支持. 1997 年, Yasui ${ }^{[6]}$ 研究 了空化泡的气液界面上的相变过程. 2003 年, Toegel 和Lohse $\mathrm{e}^{[9]}$ 进一步考虑了更多的化学反应, 其中包含 了 17 种物质的 45 种化学反应过程, 结果进一步改善 了与实验的吻合程度. 2005 年, An和Ying ${ }^{[12]}$ 整合了一 个尽量完整的模型, 考虑了除化学反应外的多种机 制, 强调了选择模型及其参数的重要性. 然而, 随着 理论模型的不断完善, 方程组规模越来越大, 模型参 数也越来越多, 理论的预见性变差. 尽管如此, 仍然 遗漏了一些新的机制. 例如, $\mathrm{Xu}$ 等人 ${ }^{[4]}$ 在实验中发现, 在含有硫酸钠粉末的浓硫酸中, 声致发光的光谱中 有时会突然出现 $589.3 \mathrm{~nm}$ 的钠原子谱线(图 1). 而浓 硫酸几乎不溶解硫酸钠, 因此, 空化泡内出现的钠谱 线只能来自硫酸钠粉末. 所以该实验表明, 包含硫酸 钠粉末的硫酸液滴进入了空化泡内部. 这是一种新的 物质交换机制，超出了已有的物理模型 ${ }^{[5 \sim 9,12]}$. 可见， 已有的基于具体物理过程的质量交换模型虽然参数众 多, 但它们仍没有真实地反映质量交换过程. 在本文 中, 我们不考虑具体的物理过程, 提出一种唯象模型, 并利用模型计算了稳定平衡半径 $R_{0}$, 得到了 $R_{0}$ 与驱动 超声的关系. 对比实验数据, 结果令人满意.

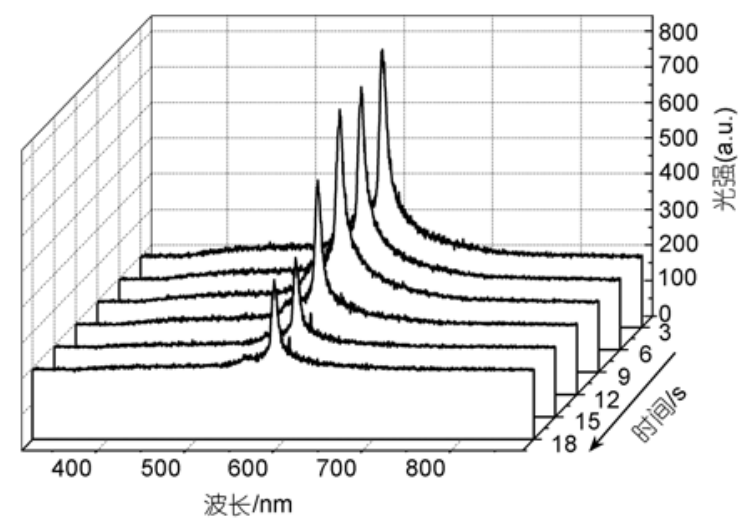

图 1 含有硫酸钠粉末的浓硫酸中氞气泡的声致发光光谱 演化

光谱中出现 $589.3 \mathrm{~nm}$ 原子谱线

1 唯象质量交换模型

文献[4]的图 7给出了 4 种情况下声致发光起始光
谱的演化过程，不同的质量交换时间尺度说明存在 气体扩散、化学反应等多种质量交换过程. 其中, 气 体扩散的主要驱动来自于物质浓度差 ${ }^{[5]}$, 气液相变和 化学反应不仅与物质浓度差有关, 还与温度等因素 有关 ${ }^{[6,7]}$. 而物质浓度差在很大程度上依赖于空化泡 内外压强差. 即使工作液体的液滴进入空化泡 ${ }^{[4]}$, 其 动力也应该主要来自于气泡内外的压强差. 因此, 我 们有理由相信, 在空化泡内外多种质量交换的过程 中, 压强差是最主要的驱动力, 是决定稳定平衡状态 的决定性因素. 所以, 我们在唯象模型中将压强差作 为空化泡内外质量交换的驱动力. 设气泡内部压强 为 $p_{\mathrm{g}}(t)$, 则空化泡内外压强差为 $\Delta p=\left(p_{\text {out }}-p_{\mathrm{g}}(t)\right)$, 其中 $p_{\text {out }}$ 是一个模型参数, 主要与空化泡外环境性质 有关. 这样, 空化泡内外质量交换可以表达为

$$
\frac{\mathrm{d} n(t)}{\mathrm{d} t}=4 \pi R^{2}(t) \delta \frac{\rho}{M}\left(p_{\text {out }}-p_{\mathrm{g}}(t)\right),
$$

其中 $n(t)$ 为 $t$ 时刻泡内物质的量(摩尔), $R(t)$ 为 $t$ 时刻气 泡半径, $M$ 和 $\rho$ 分别是工作液体的摩尔质量和密度, $\delta$ 是另一个模型参数, 它描述物质交换速率. 在第 3 节 我们将对两个模型参数具体讨论.

我们选择 Rayleigh-Plesset方程中简单经典的形 式 ${ }^{[3]}$ 来描述气泡半径的演化:

$$
\begin{aligned}
& \rho\left[R(t) \frac{\mathrm{d}^{2} R(t)}{\mathrm{d} t^{2}}+\frac{3}{2}\left(\frac{\mathrm{d} R(t)}{\mathrm{d} t}\right)^{2}\right]=\left(p_{\mathrm{g}}(t)-P_{0}-P_{\mathrm{a}} \sin \omega t\right) \\
& +\frac{R(t)}{c} \frac{\mathrm{d} p_{\mathrm{g}}(t)}{\mathrm{d} t}-\frac{4 \eta}{R(t)} \frac{\mathrm{d} R(t)}{\mathrm{d} t}-\frac{2 \sigma}{R(t)},
\end{aligned}
$$

其中 $\eta$ 和 $\sigma$ 分别为工作液体的黏滞系数和表面张力 系数. $P_{0}=1.01 \times 10^{5} \mathrm{~Pa}$ 是环境压强, $P_{\mathrm{a}} \sin \omega t$ 是我们采 用的频率 $f=\omega / 2 \pi$, 幅度为 $P_{\mathrm{a}}$ 的驱动声压.

我们采用 van der Waals 气体状态方程:

$$
\left(p_{\mathrm{g}}(t)+\frac{a n^{2}(t)}{V^{2}(t)}\right)(V(t)-n(t) b)=n(t) R_{\mathrm{g}} T(t),
$$

其中 $a$ 和 $b$ 是常量, $V(t)=\frac{4}{3} \pi R^{3}(t)$ 是空化泡的体积. 考虑能量守恒有

$$
n(t) C_{V} \frac{\mathrm{d} T(t)}{\mathrm{d} t}+\frac{a n(t)^{2}}{V^{2}(t)} \frac{\mathrm{d} V(t)}{\mathrm{d} t}=-p_{\mathrm{g}}(t) \frac{\mathrm{d} V(t)}{\mathrm{d} t}+e \frac{\mathrm{d} n(t)}{\mathrm{d} t},
$$

其中 $C_{V}$ 和 $e$ 分别是泡内气体的等容摩尔热容和摩尔 物质能量.

将(1) (4)式联立求解可得 $R(t), n(t), T(t)$ 等. 


\section{2 数值计算}

\section{1 稳定平衡半径 $R_{0}$ 和它的多重性}

数值计算表明, 在我们的可变质量模型中, 空化 泡环境半径 $R_{\mathrm{amb}}(t)$ 和物质的量 $n(t)$ 都随时间变化. 但 对合适的初始空化泡, 物质交换可以达到动态平衡. 我们定义一个周期内的净质量交换数为

$$
\Delta n=\int_{T} \frac{\mathrm{d} n(t)}{\mathrm{d} t} \mathrm{~d} t .
$$

当 $\Delta n=0$ 时, 空化泡在一个周期内进出的质量相等.

我们对任意环境半径 $R_{\mathrm{amb}}(t)$, 计算空化泡在一个 周期内的相对净质量交换数 $\Delta n /\langle n(t)\rangle$ (图 2), 其中 \langle\rangle 表 示对函数的周期平均值. 从图 2 可以看出, 曲线和横 轴有多个交点, 在这些点上 $\Delta n=0$. 在其中一些零点 上, 曲线有正的斜率, 记为 $M_{i}$; 而在另外的零点上曲 线有负的斜率, 记为 $N_{i}(i=1,2, \cdots)$. 图 2 中, $M_{i}$ 和 $N_{i}$ 将 净质量交换数曲线分成 $\mathrm{A}, \mathrm{B}, \mathrm{C}$ 区域. 在 $M_{1}$ 点的左边 $\mathrm{A}$ 区域, $\Delta n<0$, 气泡内的物质减少, $R_{\mathrm{amb}}(t)$ 沿着箭头方 向减小, $\Delta n$ 为负且绝对值越来越大, 进一步加速了物 质向空化泡外部的逃逸, 空化泡逐渐溶解于工作液 体中; 在 $M_{1}$ 点的右边 $\mathrm{B}$ 区域, $\Delta n>0$, 气泡内的物质增 多, $R_{\mathrm{amb}}(t)$ 沿着箭头方向增大, 刚开始物质进入空化 泡的速率随 $R_{\mathrm{amb}}(t)$ 增大而增大, 之后又随 $R_{\mathrm{amb}}(t)$ 增大 而减小, 直到 $N_{1}$ 点 $\Delta n=0$. 所以, 正斜率零点 $M_{1}$ 不能 稳定平衡. 在 $N_{1}$ 点右边的 C 区域, $\Delta n<0$, 气泡内物质 减少, $R_{\mathrm{amb}}(t)$ 沿着箭头的方向减小, 物质从空化泡内

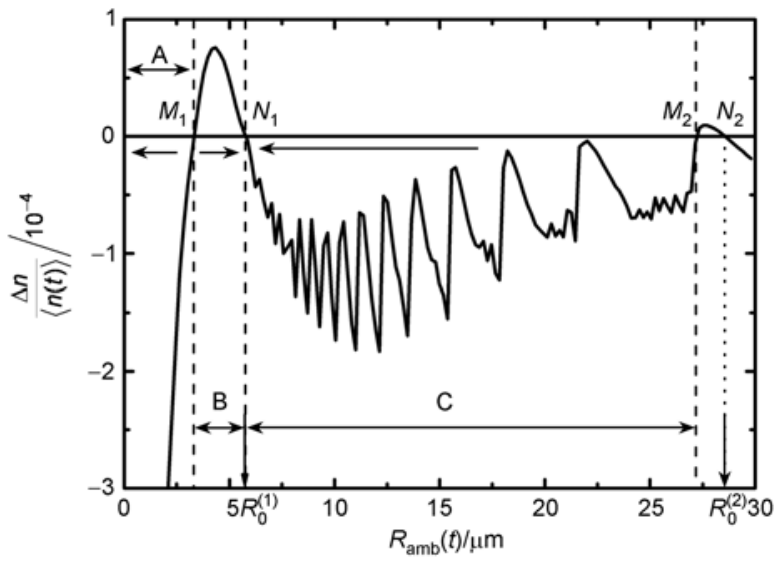

图 2 不同环境半径 $R_{\mathrm{amb}}(t)$ 的相对净质量交换数

点 $M_{i}$ 和 $N_{i}(i=1,2, \cdots)$ 使 $\Delta n=0 . M_{i}$ 和 $N_{i}$ 将图形分成 A, B, C 区域. 正 斜率零点 $M_{i}$ 不稳定平衡, 负斜率零点 $N_{i}$ 可以稳定平衡, 所以负斜 率零点 $N_{i}$ 对应稳定平衡半径 $R_{0}{ }^{(i)}$. B, C 区域的 $R_{\mathrm{amb}}(t)$ 都会稳定于 $R_{0}{ }^{(1)}, R_{0}{ }^{(2)}$ 是另外一个稳定点, 其中 $P_{\mathrm{a}}=1.20 \times 10^{5} \mathrm{~Pa}, f=25 \mathrm{kHz}$, $p_{\text {out }}=1.5 \times 10^{4} \mathrm{~Pa}$
逃逸的速率随 $R_{\mathrm{amb}}(t)$ 减小而减小, 直到 $N_{1}$ 点 $\Delta n=0$. 由此可见, 在 $\mathrm{B}, \mathrm{C}$ 区域内的 $R_{\mathrm{amb}}(t)$ 都可以收玫到负 斜率零点 $N_{1}$. 因此, 负斜率零点 $N_{1}$ 可以稳定平衡. 我 们定义平衡半径 $R_{0}$ 为负斜率零点 $N_{i}$ 处空化泡环境半 径 $R_{\mathrm{amb}}(t)$ 的周期平均值:

$$
R_{0}=\left.\left\langle R_{\mathrm{amb}}(t)\right\rangle_{N_{i}} \approx R_{\mathrm{amb}}(t)\right|_{N_{i}} .
$$

由于平衡点上 $R_{\mathrm{amb}}(t)$ 的变化很小 (图 2 中 $\Delta n /\langle n(t)\rangle \sim$ $10^{-4}$, 即 $\left.\Delta R_{\mathrm{amb}}(t) /\left\langle R_{\mathrm{amb}}(t)\right\rangle \sim 0.05\right)$, 为了节省计算量, 我 们省略积分过程, 取 $\left.R_{0} \approx R_{\mathrm{amb}}(t)\right|_{N_{i}}$ (任意时刻).

具有负斜率的零点并不唯一, 如图 2 中还有 $N_{2}$ 点, 对应平衡半径为 $R_{0}{ }^{(2)}$. 因此, 对于一定的驱动条 件, $R_{0}$ 并不是唯一的, 具有多重性, 记为 $R_{0}{ }^{(i)}, i=1,2, \cdots$. 文献[5]在理论计算中也提到过类似的结论. 从图 2 可以看出, $R_{0}{ }^{(i)}$ 的收玫区域是从之前的正斜率零点 $M_{i}$ 到其后的正斜率零点 $M_{i+1}$ 之间. 在多重平衡半径中, $R_{0}{ }^{(1)}$ 的体积最小, 形状稳定性最好, 因此实验中出现 的大部分气泡的平衡半径应为 $R_{0}{ }^{(1)}$.

在我们的实验过程中也观察到这种多重性. 在 通常的硫酸声致发光实验中, 发光的空化泡半径在 几十微米量级, 肉眼很难直接分辨. 但在一次偶然的 实验中, 在相近的实验条件下, 出现了一个肉眼明显 可见的大空化泡, 估计在亚毫米量级. 遗憾的是, 较 大的气泡通常很不稳定, 很难被测量和记录. 在下文 中, 我们以第一离散值 $R_{0}{ }^{(1)}$ 来研究平衡半径 $R_{0}$ 的相 关性质.

\section{2 与驱动声压的关系}

针对实验条件 ${ }^{[13]}$, 我们数值计算了不同声压下 空化泡的平衡半径, 图 3(a)给出了计算结果 $R_{0}-P_{\mathrm{a}}$ 关 系曲线. 从图中我们可以看到, $R_{0}-P_{\mathrm{a}}$ 曲线上存在一个 转折点, 对应的驱动声压大约为 $P_{\mathrm{a}}=1.24 \times 10^{5} \mathrm{~Pa}$, 此 时的平衡半径 $R_{0}$ 最小. 在转折点左边(低声压区), 平 衡半径 $R_{0}$ 随 $P_{\mathrm{a}}$ 增大而减小; 在转折点右边(高声压区), 它随 $P_{\mathrm{a}}$ 增大而增大. 这个 $R_{0}-P_{\mathrm{a}}$ 关系与我们已有的实 验观察一致 ${ }^{[13]}$. 而且文献[13]还发现, 这个转折点正 好对应空化泡声致发光的阈值点, 在它的左边是非 声致发光区域, 右边是声致发光区域. 在之前的数值 结果中 ${ }^{[9]}$, 也存在相似的转折点.

下面我们对这个转折过程进行深入的分析. 图 3(b)给出了随声压增大而减少的代表点(A)、随声压增 大而增大的代表点 $(\mathrm{C})$ 以及转折点附近的点 $(\mathrm{B})$ 的净 

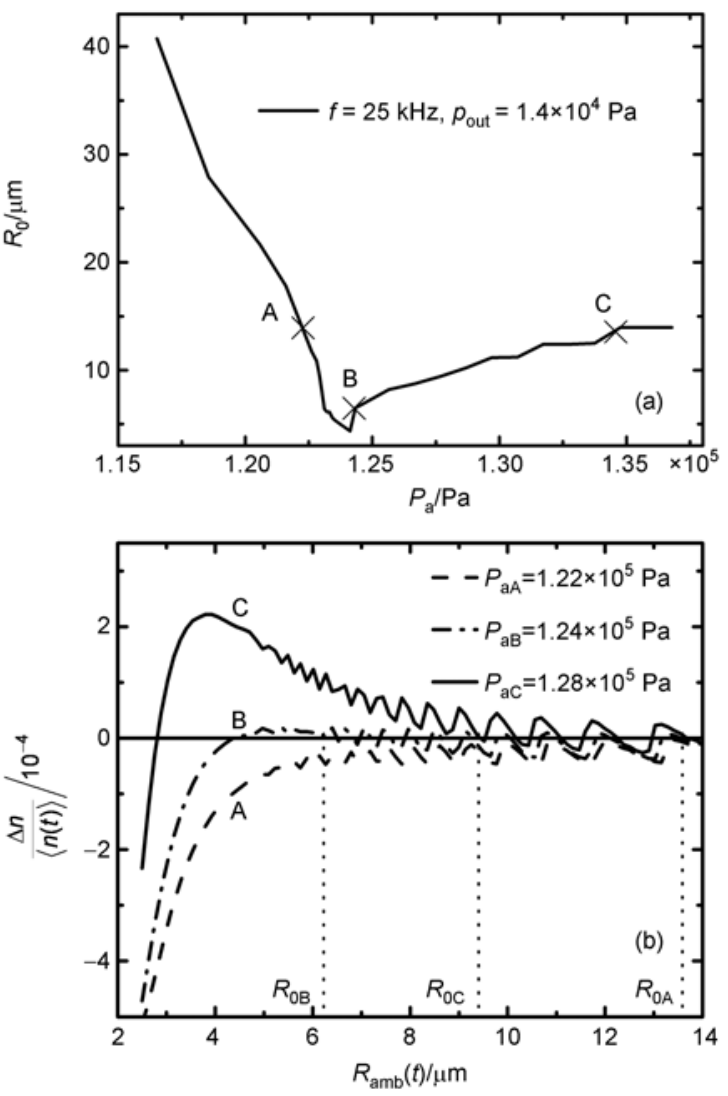

图 3 水中空化泡在不同驱动声压下的平衡半径 (a) 水中空化泡 $R_{0}-P_{\mathrm{a}}$ 曲线; (b) 相对净质量变化数曲线. $\mathrm{A}, \mathrm{B}, \mathrm{C}$ 曲 线对应的声压为由弱到强, 它们的平衡半径分别为 $R_{0 \mathrm{~A}}, R_{0 \mathrm{~B}}, R_{0 \mathrm{C}}$, 对应图(a)中“×”标注的 A, B, C 三点

质量变化数曲线. 从图 3(b)中不难看到, 随声压的增 大, 曲线头部不断上升, 导致曲线的负斜率零点左移, 这对应着图 3(a)中的低声压区平衡半径随声压增大 而减小; 当曲线上升到临界位置, 即下方的曲线头部 与横轴相切, 此时一个新的平衡位置形成; 继续增大 声压, 曲线头部继续上升, 新的负斜率零点开始右移, 对应着图 3(a)中的高声压区平衡半径随声压增大而 增大. 可见这个临界点就是对应着图 3(a)中的转折 点.

图 4 是水中空化泡在不同的驱动频率 $f$ 下的 $R_{0}-P_{\mathrm{a}}$ 曲线. 可以看出, 驱动频率 $f$ 越大, $R_{0}-P_{\mathrm{a}}$ 曲线的转折点 发生在越大的驱动声压 $P_{\mathrm{a}}$ 下．在高声压区域，驱动频 率 $f$ 越大, 空化泡 $R_{0}$ 越小. 在实验中, 我们观察到声致 发光的气泡在频率较高时的平衡半径较小. 而转折 点对应声致发光阈值点 ${ }^{[13]}$, 所以声致发光气泡的实 验结果与我们的理论结果在高驱动声压区域的

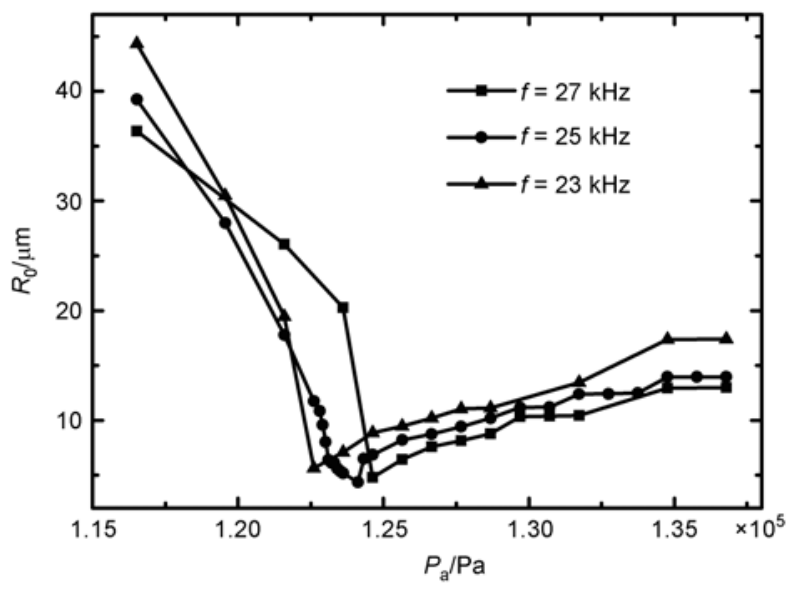

图 4 不同驱动频率下空化泡的 $\boldsymbol{R}_{\mathbf{0}}-\boldsymbol{P}_{\mathrm{a}}$ 关系曲线 工作液体为水, 其中 $p_{\text {out }}=1.4 \times 10^{4} \mathrm{~Pa}$

趋势相符.

\section{3 模型参数}

在我们的唯象质量交换(1)式中，不考虑具体的 物质交换机制, 只有两个模型参数, 即 $p_{\text {out }}$ 和 $\delta$. 下面 我们分析它们对模型计算结果的影响.

首先, 改变参数 $p_{\text {out }}$, 研究不同的 $p_{\text {out }}$ 对空化泡 $R_{0}-P_{\mathrm{a}}$ 曲线的影响, 得到的结果如图 5 所示. 在高驱动 声压区, $p_{\text {out }}$ 越大, 空化泡平衡半径 $R_{0}$ 越大; 而在低 驱动声压区, $p_{\text {out }}$ 越大，空化泡平衡半径 $R_{0}$ 则越小.

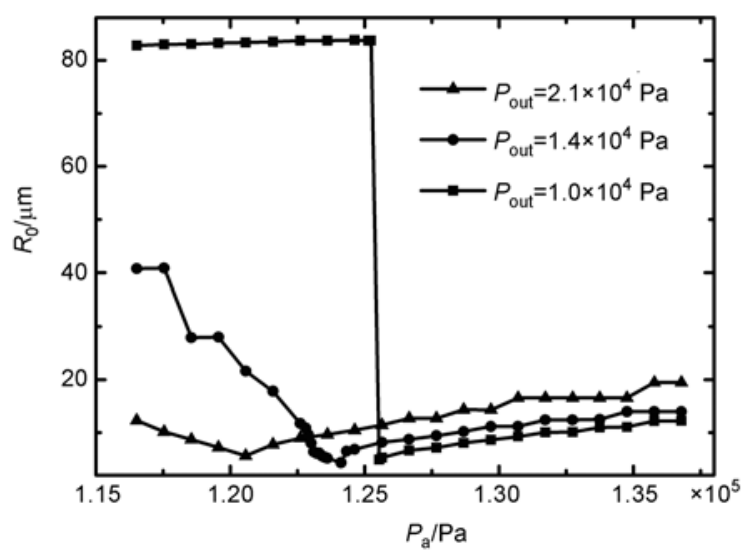

图 5 不同 $p_{\text {out }}$ 下空化泡的 $\boldsymbol{R}_{0}-\boldsymbol{P}_{\mathrm{a}}$ 关系曲线 工作液体为水, 其中 $f=25 \mathrm{kHz}$

改变 $p_{\text {out }}$ 的大小, 还会影响 $R_{0}-P_{\mathrm{a}}$ 曲线的转折点 位置. 减小 $p_{\text {out }}$ 导致曲线及其转折点右移, 即发生的 驱动声压 $P_{\mathrm{a}}$ 变大. 从(1)式可知, 减小 $p_{\text {out }}$ 可以使空化 泡的净质量交换数趋于负值. 当空化泡进行非线性 
振动时, 增大 $P_{\mathrm{a}}$ 可以提高气泡内部气体的周期平均 压强 $\left\langle p_{\mathrm{g}}(t)\right\rangle$, 使空化泡的净质量交换数恢复为零, 从 而达到新的平衡. 同时, 对于小的 $p_{\text {out }}$, 在低声压区 曲线出现平衡半径跳跃的现象(图 5 中“口”, $p_{\text {out }}=$ $1.0 \times 10^{4} \mathrm{~Pa}$ ). 所谓平衡半径的跳跃是指气泡大小极度 依赖于驱动声压. 一个小的驱动声压变化, 会导致空 化泡平衡半径量级的变化. 显然, 这样的气泡在实验 上是不易观察的.

$p_{\text {out }}$ 是描述泡外环境性质的综合参数, 它与气体 扩散过程中的液体含气量及化学反应中的物质浓度 等有关. 由于是个综合参数, 它的值不能通过实验直 接测量, 只能通过空化泡半径演化曲线和气泡动力 学方程拟合获得 $R_{0}$ 值来确定. 我们对 $p_{\text {out }}$ 和液体含气 量的参数相关性进行了简单的实验研究, 发现在保 持其他参数不变的情况下, 工作液体含气量越高, 相

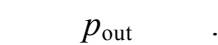

模型参数 $\delta$ 是一个描述空化泡内外质量交换速率 的参数, 是扩散系数、气液相变快慢及化学反应速率 等综合的结果. 它与实验中测得的质量交换的时间 标度有关. 时间标度越大，对应的 $\delta$ 值越小，反之亦 然. 在实验 ${ }^{[4]}$ 中, 我们通过测量起始声致发光的光谱 演化，得到质量交换的时间标度，可以由此确定 $\delta$ 的 大小. 然而我们在数值计算中发现, $\delta$ 在相当大的范 围 $\left(\delta<1.0 \times 10^{-13} \mathrm{~m}^{3} /(\mathrm{N} \cdot \mathrm{s})\right)$ 内变化, 除了改变质量交换 的时间标度, 几乎不影响最终的平衡半径的大小. 考 虑到本文旨在获得不同驱动条件下平衡半径的大小, 所以在计算中为了节省计算量, 可以尽量选取较大 的 $\delta$, 我们这里取 $\delta=1.0 \times 10^{-14} \mathrm{~m}^{3} /(\mathrm{N} \cdot \mathrm{s})$. 当然, $\delta$ 过 大会导致物质逃逸过快等非物理现象的出现.

\section{4 实验和理论的比较}

我们用文献[13]中所述的锁相积分拍摄成像技 术，测量了硫酸中稳定悬浮声致发光气泡的半径时 间演化曲线，通过与 Rayleigh-Plesset方程进行拟合， 得到平衡半径. 然后改变驱动声压, 得到了硫酸中 $R_{0}-P_{\mathrm{a}}$ 曲线的实验数据(图 6 中“口”所示). 然后, 我们在 唯象模型中输入硫酸的参数, 调整模型参数 $p_{\text {out }}$, 得 到了相应的理论 $R_{0}-P_{\mathrm{a}}$ 曲线 (图 6 中“.”所示). 由于 $R_{0}-P_{\mathrm{a}}$ 曲线的转折点对应于声致发光的阈值点 ${ }^{[13]}$, 所 以, 声致发光气泡的实验数据全部落在我们数值结 果的高驱动声压区. 实验和高声压区的理论结果符 合得很好, 并且都显示出平衡半径 $R_{0}$ 随驱动声压 $P_{\mathrm{a}}$
的增大而增大 (图 6). 遗憾的是, 在实验中当 $P_{\mathrm{a}}<$ $1.34 \times 10^{5} \mathrm{~Pa}$ 时, 硫酸中的空化泡出现不稳定性, 无法 悬浮，因而我们没有获得低声压区的实验数据.

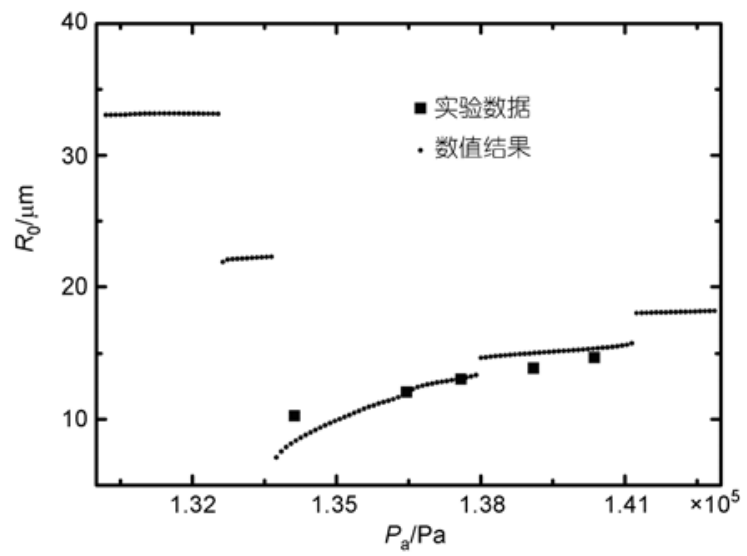

图 6 硫酸中空化泡的 $R_{0}-P_{\mathrm{a}}$ 曲线的实验和理论比较 $f=26.5 \mathrm{kHz}$, 模型参数 $p_{\text {out }}=2.5 \times 10^{4} \mathrm{~Pa}$

\section{5 结论}

本文提出了一种描述空化泡内外质量交换的唯 象模型. 我们抓住空化泡内外质量交换的主要驱动 因素一一压强差来建立方程. 联合Rayleigh-Plesset气 泡动力学方程, 我们得到了空化泡在给定驱动声压 下的稳定平衡半径, 并研究了平衡半径的多值性. 水 中 $R_{0}-P_{\mathrm{a}}$ 曲线的数值结果与实验结果 ${ }^{[13]}$ 相吻合: 在低 驱动声压区, 平衡半径随驱动声压增大而减小, 在高 驱动声压区, 平衡半径随驱动声压增大而增大. 中间 存在一个转折点, 即声致发光的阈值点 ${ }^{[13]}$. 我们还研 究了模型参数 $p_{\text {out }}$ 和 $\delta$ 对 $R_{0}-P_{\mathrm{a}}$ 曲线的影响: 减小 $p_{\text {out }}$ 可 以使 $R_{0}-P_{\mathrm{a}}$ 曲线及其转折点右移, 较小的 $p_{\text {out }}$ 使低声压 区域产生平衡半径跳跃的现象; 而改变 $\delta$ 只影响空化 泡内外质量交换的时间标度, 几乎不影响最后的平 衡半径的大小. 最后, 我们实验测量了不同驱动声压 下的硫酸中空化泡的半径演化曲线, 得到实验 $R_{0}-P_{\mathrm{a}}$ 曲线. 理论和实验的良好一致性证明了我们的唯象 模型的有效性.

可见，本文提出的唯象质量交换模型具有直观 简单、计算量少的特点, 它能合理地描述极为复杂的 气泡内外质量交换过程. 对模型的进一步完善可以 提高模型结果与实验数据的定量吻合程度, 更好地 解释气泡质量交换现象，预测更多的相关性质. 


\section{参考文献}

Leighton T G. The Acoustic Bubble. London: Academic Press, 1994

Rayleigh L. On the pressure developed in a liquid during the collapse of a spherical cavity. Phil Mag, 1917, 34(6): 94-98

Brenner M P, Hilgenfeldt S, Lohse D. Single-bubble sonoluminescence. Rev Mod Phys, 2002, 74(2): 425—477미]

$4 \mathrm{Xu} \mathrm{J} \mathrm{F}$, Chen W Z, Xu X H, et al. Composition and its evolutions inside a sonoluminescing bubble by line spectra. Phys Rev E, 2007, 76(2): 026308ㅁDㅁ]

5 Hilgenfeldt S, Lohse D, Brenner M P. Phase diagrams for sonoluminescing bubbles. Phys Fluids, 1996, 8(11): 2808 — 2826[DOI]

6 Yasui K. Alternative model of single-bubble sonoluminescence. Phys Rev E, 1997, 56(6): 6750-6760[DOI]

7 Lohse D, Hilgenfeldt S. Inert gas accumulation in sonoluminescing bubbles. J Chem Phys, 1997, 107(17): 6986-6997[DOI]

8 Lohse D, Brenner M P, Dupont T F, et al. Sonoluminescing air bubbles rectify argon. Phys Rev Lett, 1997, 78(7): 1359-1362[DOI]

9 Toegel R, Lohse D. Phase diagrams for sonoluminescing bubbles: A comparison between experiment and theory. J Chem Phys, 2003, 118(4): 1863-1875[DOD]

10 Matula T J, Crum L A. Evidence for gas exchange in single-bubble sonoluminescence. Phys Rev Lett, 1998, 80(4): 865-868[DOI]

11 Ketterling J A, Apfel R E. Experimental validation of the dissociation hypothesis for single bubble sonoluminescence. Phys Rev Lett, 1998, 81(22): 4991-4994 [DOI]

12 An Y, Ying C F. Model of single bubble sonoluminescence. Phys Rev E, 2005, 71(3): 036308[DOI]

13 Huang W, Chen W Z, Liu Y N, et al. The evolution of the cavitation bubble driven by different sound pressure. Ultrasonics, 2006, 44: e407-e410 $\underline{\text { DOI] }}$

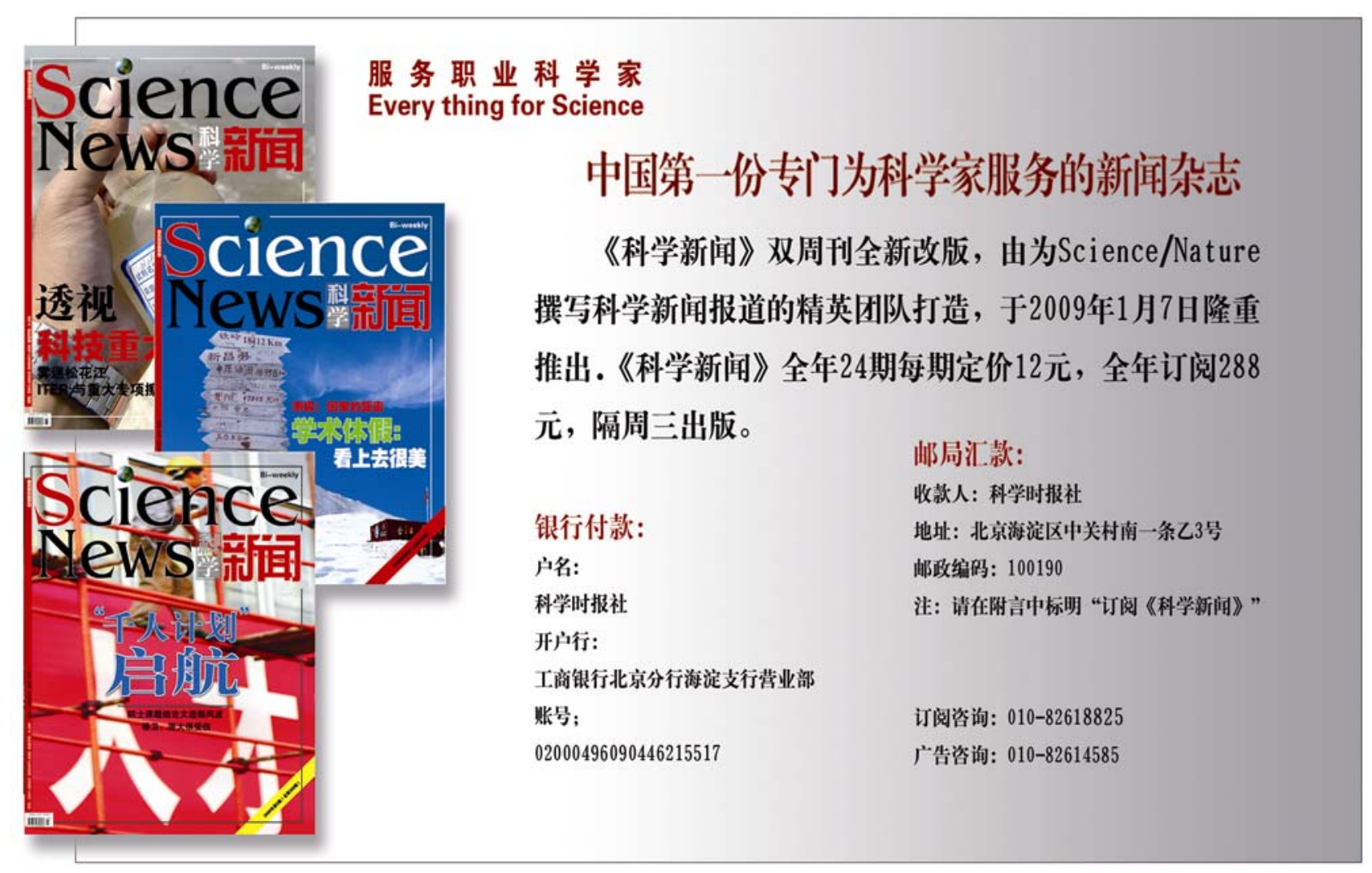

